\title{
The Absence of Blocking Innicotine High-Responders as a Possible Factor in the Development of Nicotine Dependence?
}

\author{
Adi Jaffe ${ }^{*}, 1$ J. Aurora Z. Pham ${ }^{1}$, Igal Tarash ${ }^{1}$, Sasha S. Getty ${ }^{1}$, Michael S. Fanselow ${ }^{1}$ and \\ J. David Jentsch ${ }^{1,2}$
}

Departments of ${ }^{1}$ Psychology and ${ }^{2}$ Psychiatry and Bio-behavioral Sciences, University of California, Los Angeles, USA

\begin{abstract}
Rationale: The importance of reward-associated cues in eliciting behavior is well established, with stimuli associated with drugs of abuse known to play a crucial role in recidivism. Recently, Redish (2004) proposed that drugs, acting as unconditional stimuli (US), remain associable even after being fully predicted by a conditional stimulus (CS), meaning that they are not susceptible to the blocking effect [1]; if correct, this may represent a possible mechanism to explain exaggerated cue-controlled drug-seeking and reinstatement in nicotine dependence and substance dependence problems in general.

Objectives: We tested whether pairings between nicotine and an environmental CS would convey conditioned reinforcement properties onto the CS, even when nicotine's rewarding effects were already fully predicted by another cue (whether there was an absence of the blocking effect).

Methods: 134 male Long-Evans rats were implanted with jugular catheters and assigned to either food- or nicotine-reward $(0.06 \mathrm{mg} / \mathrm{kg} / \mathrm{inf})$ conditions. Each group was exposed to paired or unpaired presentations of their respective reward with one CS in 10 daily sessions; subsequently, they were exposed to 4 more daily sessions of paired presentations of the reward paired with a compound CS composed of the original CS and a second CS. Tests of the conditioned reinforcing value of both CSs using the active-lever-presses to total-presses ratio as an outcome were conducted following training.

Results: Pressing for a blocked second CS $(\mu=0.59, S D=0.21)$ was significantly lower than pressing for an unblocked second CS $(\mu=0.69, S D=0.14)$ in the food-reward condition, but not in nicotine-rewarded animals, verifying the hypothesis that nicotine, but not food, is resilient to the blocking effect.

Conclusion: The absence of blocking when nicotine is the reward may explain the powerful role for cues in supporting tobacco dependenceby allowing for the extension of nicotine's rewarding value across numerous associated cues.
\end{abstract}

Keywords: Addiction, blocking effect, conditioned reinforcement, dependence, nicotine, pavlovian, tobacco.

\section{INTRODUCTION}

Drug abuse has become one of the leading social ills of our modern society, due, in large part, to its impact on the physical and mental health of the individual with consequential effects on families and society at large. Dependence on tobacco is quite possibly the most devastating drug abuse problem, given its prevalence throughout the world and the resultant health issues. Over $12 \%$ of the world's mortality is attributable to tobacco use with estimates of approximately 5 million deaths annually being caused by cigarette smoking $[2,3]$. One of the most vexing aspects of tobacco dependence is its incredibly persistent nature, even in the face of obvious, and often debilitating, negative consequences.

Nicotine is considered to be the primary addictive substance in tobacco, as nicotine acts as a reinforcer in laboratory animals and humans, especially following preexposure to the drug [4-8]. Still, nicotine's reinforcing

*Address correspondence to this author at the Departments of Psychology and Psychiatry and Bio-behavioral Sciences, University of California, Los Angeles, USA; Tel: (310) 488-3978; E-mail: adi@ucla.edu properties have repeatedly proven to be relatively weak and to depend greatly upon predictive cues that support its reinforcing effects $[8,9]$. Therefore, some researchers have proposed that nicotine's addictive potential lies not only in its primary reinforcing properties, but also in its ability to enhance the reinforcing value of other events and rewards $[10,11]$. Furthermore, findings regarding the disparate extended response to nicotine-associated cues in "responders" versus "non-responders shown by Cohen and colleagues [12] suggest that examinations regarding nicotine's addictive potential need to consider individual differences in reinforcement-response to the drug itself.

Undoubtedly, nicotine dependence presents a puzzling inconsistency in which the world's most commonly abused substance has been found to be only weakly reinforcing. It is almost certain that the tobacco's legal status, as well as its wide availability and prominent role in society, are important factors in its broad abuse, though other factors are likely at play.

The purpose of this study was to examine whether the resolution of this apparent paradox may lie in the effect of nicotine over a learning mechanism known as the blocking 
effect, which limits the associability of cues with a given reward $[1,13,14]$. For positive reinforcers like food and drugs, blocking is thought to depend on "error-detection"related phasic-discharge activity in dopaminergic neurons; unexpected natural rewards elicit this discharge, which may induce learning, while already fully predicted (i.e., fully and reliably associated with preceding cues) natural rewards no longer cause dopaminergic firing [15]. Because some drugs of abuse act as rewards and reinforcers through direct pharmacological modulation of dopaminergic neurons, even after fully predicted, it is possible that they remain associable to a larger number of predictive stimuli [16], possibly explaining the exaggerated role of drug-associated cues in directing, as well as reinstating, drug-seeking and drugtaking behavior in both animals and humans [17-22]. Based upon this hypothesis, Panlilio et al. examined the behavioral significance of drug-associated cues in a rodent cocaine selfadministration/cue-induced reinstatement model; they reported that cocaine was susceptible to the blocking effect, in contrast to the hypothesis [23]. That said, cocaine does not increase the error-related discharge activity of dopaminergic neurons, which is dependent on phasic release; as a reuptake inhibitor, it is, in fact, known to inhibit dopaminergic neuron firing due, in-part, to its activation of presynaptic D2 autoreceptors. On the other hand, nicotine does enhance phasic dopamine (DA) activity in the midbrain-NAc pathway, through its activation of nicotinic receptors in the ventral tegmental area (VTA); [24-26]. Given the apparently phasic nature of the DA-dependent "error-detection" signal [27-30], it is possible that the pharmacological effect of nicotine on DA would create a disruption in the blocking effect, a possibility we tested using a two-phase Pavlovian blocking procedure.

Numerous previous pre-clinical findings have shown that animals responding for food and a number of drugs of abuse, including amphetamine, nicotine, and cocaine, can often be grouped into low, and high, responding subgroups on the basis of measures as varied as locomotor activity, lever pressing, delay-discounting, and drug-seeking during abstinence [31-34] For this reason, we investigated the possibility of the blocking effect being disrupted in nicotinerewarded animals both in the entire sample and for animals that displayed a specifically increased response to food- and nicotine-reward. Given the relatively limited prevalence of compulsive drug-taking and drug-dependence among individuals who have ever been exposed to a specific drug and the fact the numerous factors have been shown to alter human response to nicotine, it was expected that differential effects will be revealed using such a multiple group analysis $[35,36]$.

\section{METHODS}

All procedures were previously approved by the UCLA Chancellor's Animal Research Committee (protocol \#2007132). All subjects were maintained under conditions consistent with policies of the University of California, Los Angeles, Public Health Service and the Society for Neuroscience.

\section{Animals}

Subjects were adult male Long-Evans rats (Harlan, Indianapolis IN), weighing 250-300 g at receipt. Animals
$(N=134)$ were housed in pairs in a temperature- controlled environment under 14: 10-hr light-dark conditions. Food availability was restricted after the post-surgical recovery period in order to maintain the subjects at $\sim 90 \%$ of their freefeeding weights.

\section{Apparatus}

All experimental procedures were conducted in eight aluminum and Plexiglas chambers $(25 \times 31 \times 24 \mathrm{~cm}$; Med Associates, St. Albans, VT), each housed in a soundattenuated cubicle (Med Associates, St. Albans, VT). Each box was constructed of Plexiglas front and back walls, a metal panel fitted with two nose-poke apertures, each equipped with three cue lights, and two retractable levers, all located approximately $3 \mathrm{~cm}$ from the grid floor of the chamber. On the opposite panel was photocell-equipped receptacle for delivery of $45 \mathrm{mg}$ Dustless precision pellets (Bio-serv Inc., Allentown NJ). The boxes were also equipped with a white noise generator, a SONalert tone generator and a house light. Infusions (inf) were delivered using an automatic pump connected by a freely rotating tether to a top mounted swivel that allowed virtuallyunrestricted movement. All test session functions were controlled by a computer using Med Associates Med PC IV software and interface (Med Associates, St. Albans, VT).

\section{Intravenous Catheterization Surgery}

All rats were anesthetized using an isoflurane $(2.5 \%)$ /oxygen mixture, treated with a non-steroidal analgesic (carprofen; $5.0 \mathrm{mg} / \mathrm{kg}$ ) and prepared with a catheter inserted into the right jugular vein. Catheters were prepared by the lead author using methods previously described by Markou and Paterson (2001). Animals were given 7-10 days to recover from surgery, during which time they continued to receive a non-steroidal analgesic (carprofen; $5.0 \mathrm{mg} / \mathrm{kg}$ ) and daily administration of a $0.2 \mathrm{~mL}$ infusion of Timentin and heparin solution $(100 \mathrm{mg} / \mathrm{mL}$ timentin and $33.3 \mathrm{U} / \mathrm{mL}$ heparin) to promote catheter patency.

\section{Nicotine Treatment}

Subjects in the drug- reward condition were administered nicotine $(0.06 \mathrm{mg} / \mathrm{kg} / \mathrm{inf})$, which was prepared by dissolving nicotine hydrogen tartrate (Sigma-Aldrich) in saline $(0.9 \%)$ and adjusting the $\mathrm{pH}$ to 7.4. The solution was administered using programmed 2-s infusions (Razel Model A; Stamford $\mathrm{CT})$ at appropriate intervals during the conditioning procedure via the implanted jugular catheter. Nicotine dose and administration procedures are consistent with previous studies of nicotine self-administration in rodents [37, 38].

\section{Procedures}

After recovery from surgery, animals were randomly assigned to one of four different conditions, as shown in Fig. (1.1); the four groups were derived from a $2 \times 2$ design in which they received either nicotine infusions or food pellets as the US and the 1st phase cue was either paired or unpaired with the US. All animals received pairings of the $2^{\text {nd }}$ phase cue with the US.

During phase 1 of the experiment, rats were exposed to 10 -s presentations of their $1^{\text {st }}$ phase experimental CS (i.e., overhead light or pulsing tone; cues were counterbalanced 

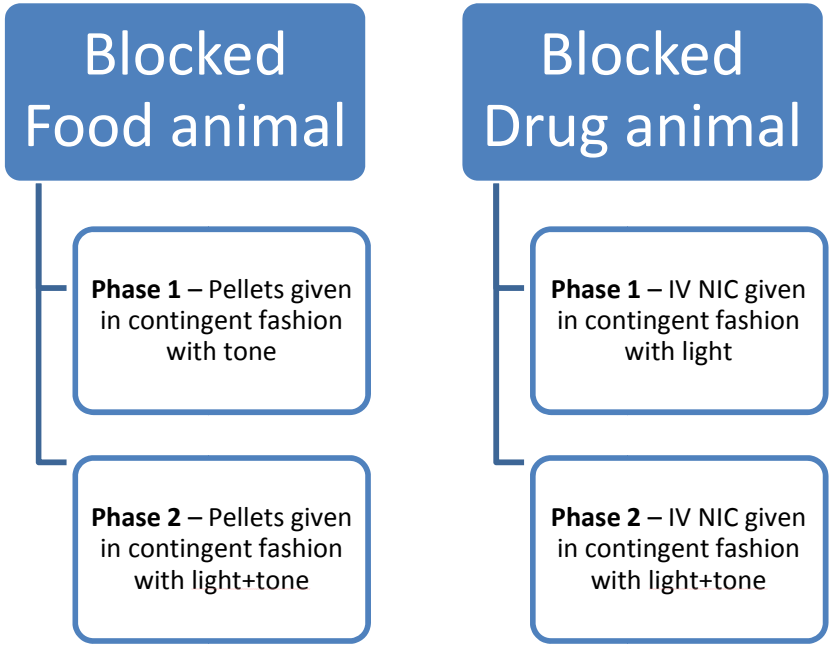

Fig. (1). A schematic of the four experimental conditions.

across and within conditions) that were paired, or unpaired, with reward delivery. In the paired, food-reward condition, pellets ( 2 at a time) were dropped into the magazine on a random time (RT)-5-s schedule, except during the first or last second of the CS. This resulted in an average of two food deliveries per 10-s CS presentation interval. The latency from CS onset to food-magazine head-entry was recorded. Each presentation interval was followed by a pseudo-random variable ITI programmed to emulate nicotine self-administration with a loading period (60", 90", 150", 210", 210", 240", 270", 270", 300", 300”, 330", 330”, 360", $360 ", 360$ "). For drug-rewarded animals, a similar procedure was employed, replacing food-pellet delivery with programmed 2-s nicotine infusion, which began immediately upon CS onset. In the unpaired condition, CS presentations were delivered during the timeout period, again using a pseudo-random schedule (30", 60", 100", 150", 150", 200", $220 ", 220 ", 250 ", 250 ”, 280 ", 280 ", 300 ", 300 ", 300 ”)$. For all groups, a total of 15 CSs were presented during each 65min session. Daily sessions were continued for 10 days, at which point Phase 1 was complete and procedures for phase 2 of the experiment began.

In phase 2 , all animals had a $2^{\text {nd }}$ phase cue (i.e., overhead light or pulsing tone, whichever was not the $1^{\text {st }}$ phase CS) introduced into the sessions. This cue, serving as the $2^{\text {nd }}$ phase CS, was always paired with reward delivery, while 1st phase CS presentations continued in the same Phase 1 condition-appropriate manner. This resulted in a compound stimulus cue for animals in the blocked condition. Training in phase 2 continued for 4 days and each daily session consisted of $15 \mathrm{CS} / \mathrm{US}$ presentations.

\section{Conditioned Reinforcement (CR) Testing}

Conditioned reinforcement tests were administered on the two days following completion of training. Test sessions were timed to last $30 \mathrm{~min}$ from the first active CR lever response (see Taylor and Horger 1999). The first test assessed the CR properties of the $2^{\text {nd }}$ phase CS; for this test the location of the active lever (i.e., Right or Left) was counterbalanced across subjects. Active lever presses initiated a 5-s presentation of the $2^{\text {nd }}$ phase CS. On the following day, a similar testing session was undertaken

\section{Unblocked Food animal}

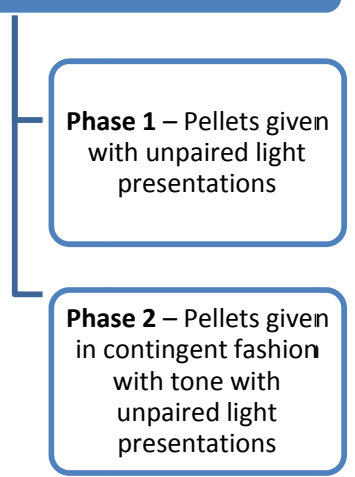

\section{Unblocked Drug animal}

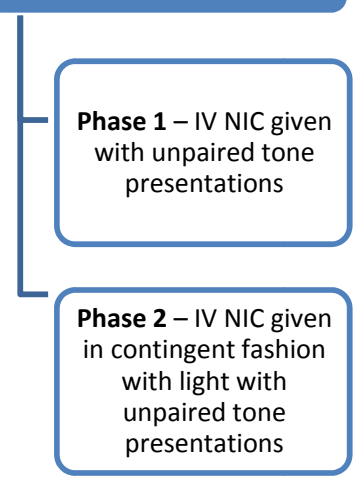

assessing CR for the $1^{\text {st }}$ phase, or initial, CS. Active lever assignment for the second CR test was retained from the initial testing session in order to control for lever effect.

\section{Statistical Methodology}

For data from the $\mathrm{CR}$ tests, the $1^{\text {st }}$ phase dependent variables were created by dividing the number of active lever presses by total lever presses (i.e., active + inactive presses). These variables are referred to as the 1st phase- and 2nd phase-Total-Ratios. The measures were analyzed using a Multivariate ANOVA (MANOVA) using Pairing (Paired vs Unpaired) and Reward (Food vs Nicotine) as independent variables. Data entry and analysis was performed using SPSS 13, and Alpha levels of 0.05 were used throughout as the standard for rejection of the null hypothesis.

\section{RESULTS}

Our initial hypothesis that the blocking effect would not be observed for animals in the nicotine-reward group required the assessment of several indicators of conditioning. Results from Phase I of our experiment needed to establish that our conditioning procedure, when applied to both nicotine-, and food-rewarded, animals, would produce the learning necessary for blocking to be possible in Phase II. It is only at that point that learning, or lack thereof, with reference to that $2^{\text {nd }}$ phase cue could be taken as evidence of blocking to its absence. For this reason we present our results in the same order as the conditioning procedures mentioned earlier.

Phase I analysis - We conducted a 2 (paired vs unpaired) by 2 (food $v s$ drug) ANOVA using the ratio of active-lever presses to the total number of lever presses as our dependent variable (Column A, Fig. 2). We found significant main effects for pairing $(\mathrm{F}(1,117)=21.06$, $\mathrm{p}<0.001$, partial $\eta 2=0.15)$ and reward $(\mathrm{F}(1,117)=6.71$, $\mathrm{p}<0.05$, partial $\eta 2=0.05)$, with a non-significant interaction between the two factors $(\mathrm{F}(1,117)=1.24$, ns, partial $\eta 2=0.01)$. Given that our hypothesis regarding blocking required that learning about the $1^{\text {st }}$ phase CS occur for the both food- and nicotine-rewarded animals, we followed up the omnibus analysis with individual analyses of each reward group. As expected, CR properties of the cue 


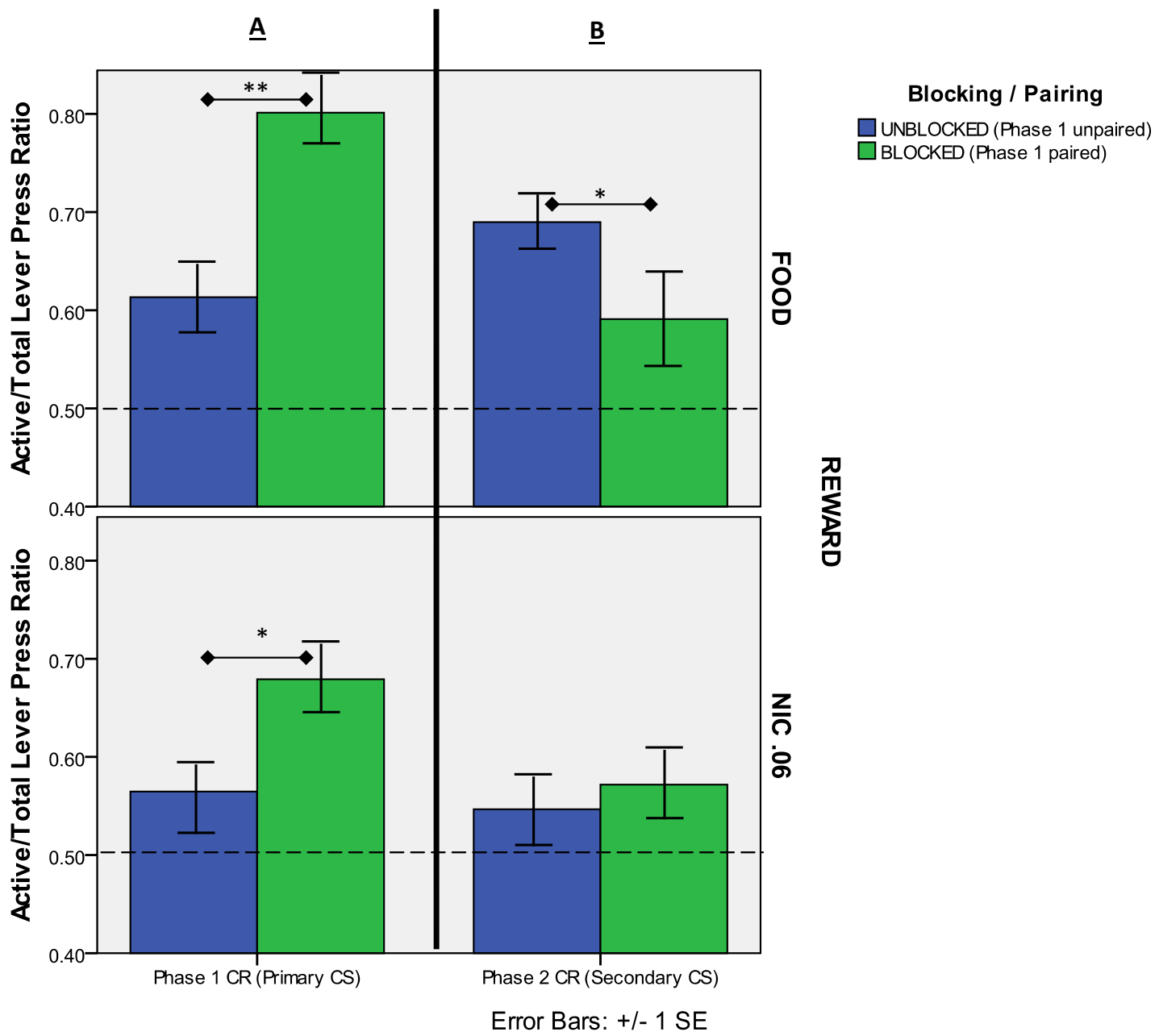

Note: The dashed line represents the point at which active presses = inactive presses $* p<0.05$

$* * p<0.01$

Fig. (2). Active-lever to total-press ratio for primary and secondary CR tests.

were significantly higher in the food-rewarded condition among paired-CS $(\mu=0.80, \mathrm{SD}=0.12)$ versus unpaired-CS $(\mu=0.61, \mathrm{SD}=0.15)$ animals $(\mathrm{F}(1,56)=26.89, \mathrm{p}<0.001$, partial $\eta 2=0.32$ ). This finding was bolstered by our analysis of the retrieval latencies in food-rewarded animals which showed that although both groups displayed similar latencies on day 1 of training $(\mathrm{t}(28)=0.071$, ns), the group receiving paired $\mathrm{CS}+$ food pairings showed reductions in magazine entry latencies with subsequent training (Fig. 3). This difference became statistically significant on day 2 of training $(\mathrm{t}(28)=3.40, \mathrm{p}<0.01)$ and remained significant throughout training. The unpaired group did not exhibit similar signs of cue conditioning.

A similar pattern of results, yielding a noticeably smaller effect size, was found in the nicotine-reward condition with paired-CS $(\mu=0.68, S D=0.21)$ animals exhibiting a significantly greater $\mathrm{CR}$ effect compared to the unpaired-CS $(\mu=0.56, \mathrm{SD}=0.21)$ animals $(\mathrm{F}(1,61)=4.55, \mathrm{p}<0.05$, partial $\eta 2=0.07)$, showing that the 1 st phase cue served as a conditioned reinforcer in animals exhibiting paired, but not unpaired, presentations of nicotine and the $1^{\text {st }}$ phase cue.
To further describe these results, we conducted analyses comparing Phase I empirical results to a hypothetical "Null Learning" condition where the mean ratio would have been equal to 0.5 (Table 1). Both paired conditions (i.e., food- and nicotine-reward) yielded significantly greater ratios than 0.5 , while the same was true only for food-rewarded, but not nicotine-rewarded, animals in the unpaired conditions.

Phase II analysis - Having established learning for the 1st phase CS under both reward conditions, we next analyzed results for the $2^{\text {nd }}$ phase CS using a similar analytic strategy.

A 2 (Paired $v s$ Unpaired) x 2 (food $v s$ drug) ANOVA for the $\mathrm{CR}$ properties of the 2 nd phase cue was performed (Column B, Fig. 2). We found a significant main effect of reward $(F(1,117)=5.31, p<0.05$, partial $\eta 2=0.04)$, but no main effect for pairing $(\mathrm{F}(1,117)=1.09$, ns, partial $\eta 2=$ $0.01)$. The interaction between pairing and reward reached the trend level $(F(1,117)=3.09, p=0.08$, partial $\eta 2=0.03)$. As the $1^{\text {st }}$ phase test of the hypothesis involved a contrast between food and nicotine conditions, we analyzed the 2 nd phase CR results separately for the food- and nicotine- 
Table 1. 1 sample t-test results assessing CR performance differences from a hypothetical "no learning" result where the ratio would equal 0.50 .

\begin{tabular}{|c|c|c|c|c|c|c|}
\hline & $\mathbf{N}$ & & Mean & S.E. & $\mathbf{t}$ & p \\
\hline \multirow{2}{*}{ Food - Unblocked } & \multirow{2}{*}{26} & Phase 1 & .61 & .028 & 6.68 & $<0.001$ \\
\hline & & Phase 2 & .69 & .03 & 3.72 & $=0.001$ \\
\hline \multirow{2}{*}{ Food - Blocked } & \multirow{2}{*}{32} & Phase 1 & .80 & .021 & 14.1 & $<0.001$ \\
\hline & & Phase 2 & .59 & .038 & 2.42 & $n s$ \\
\hline Nicotine - Unblocked & 34 & Phase 2 & .55 & .035 & 1.35 & $n s$ \\
\hline \multirow{2}{*}{ Nicotine - Blocked } & \multirow{2}{*}{29} & Phase 1 & .68 & .039 & 4.61 & $<0.001$ \\
\hline & & Phase 2 & .57 & .036 & 1.97 & $n s$ \\
\hline
\end{tabular}

Note: Given the large number of comparisons in this analysis, an alpha level of 0.01 was used to determine significance.

reward groups. As expected, food-rewarded animals provided more active-lever presses in the non-blocked (unpaired 1st phase cue) condition $(\mu=0.69, \mathrm{SD}=0.14)$ than in the blocked (paired 1st phase cue) condition $(\mu=0.59, \mathrm{SD}=0.21)$, demonstrating blocking $(\mathrm{F}(1,56)=$ $4.05, \mathrm{p}<0.05$, partial $\eta 2=0.07)$. On the other hand, among nicotine-rewarded animals, slightly more active-lever presses were provided in the blocked $(\mu=0.57, \mathrm{SD}=0.20)$ than in the non-blocked $(\mu=0.55, \mathrm{SD}=0.20)$ group, though this difference was not significant, $\mathrm{F}(1,61)=0.249$, ns, partial $\eta 2=0.004$

As above, we conducted analyses comparing Phase II results to a hypothetical "Null Learning" result condition. In Phase II, the non-blocked food condition, in which the $2^{\text {nd }}$ phase CS was delivered contingently with food-pellets, provided a significantly greater ratio of active to total lever presses than the "Null Learning" hypothetical, while the blocked group did not, demonstrating the blocking effect. On the other hand, at the group level, neither blocked, nor non-

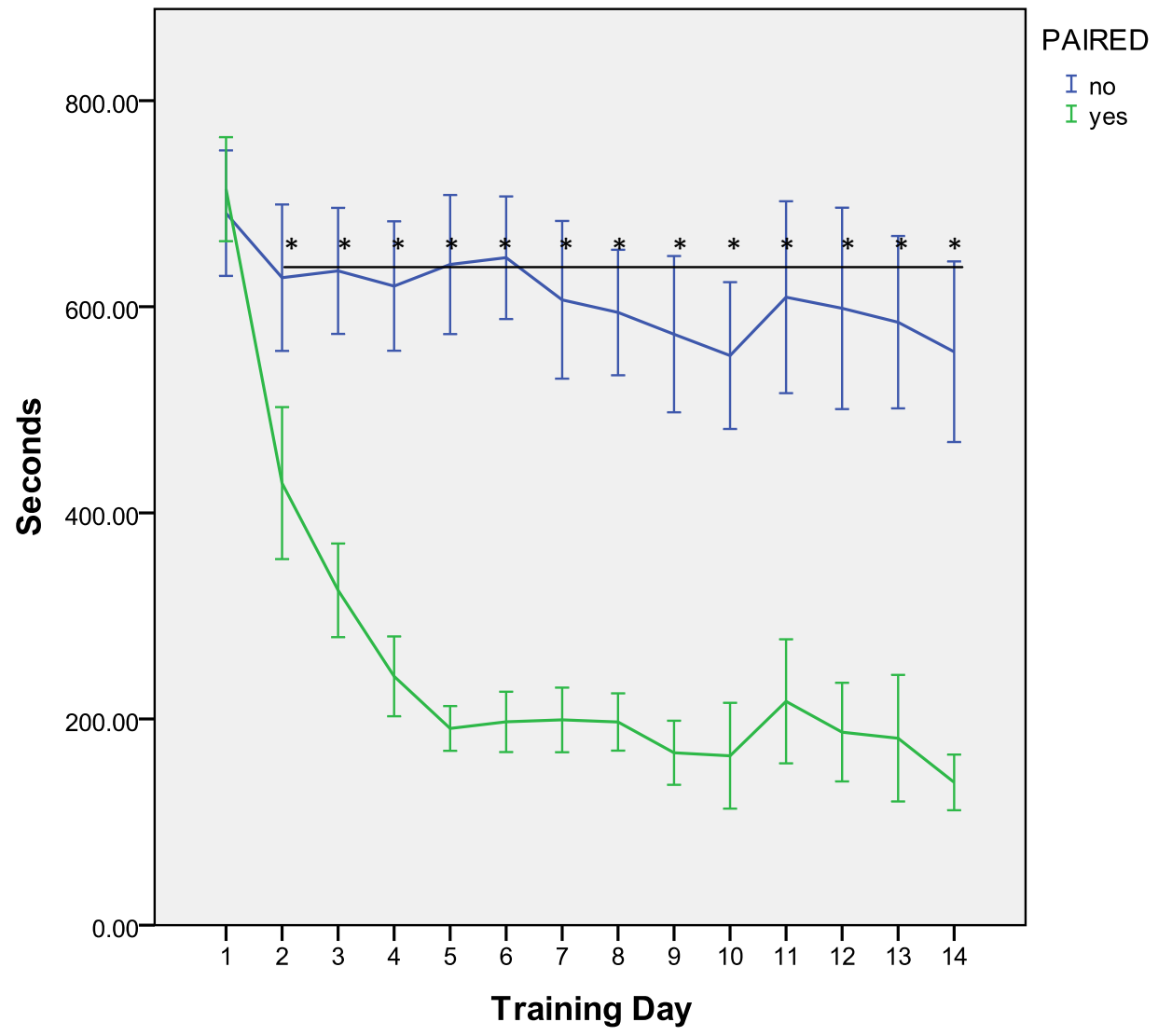

${ }^{*} p<0.01$, Error-bars designate $95 \%$ confidence-interval range

Fig. (3). Food-Magazine entry latencies (in seconds). 
blocked, nicotine-rewarded animals exhibited significant CR for the 2nd phase cue.

High-CR analysis - The previous analyses revealed a distinction in CR performance between food- and nicotinerewarded animals, especially in terms of responding for the $2^{\text {nd }}$ phase (i.e., blocked or non-blocked) CS. Given the relatively weak reinforcing value often found in nicotine experiments, and the large variability in CR performance among nicotine-rewarded subjects, we followed these analyses with a similar set focused specifically on subjects that provided relatively large CR performance. Those subjects exhibiting the highest responding for the nicotinepaired 1st phase cue were selected for further analysis; only those subjects in the highest quartile for this measure were included (Fig. 4). These subjects $(\mathrm{N}=7)$ definitionally exhibited higher 1 st phase CS responding $(\mu=0.91, \mathrm{SD}=$ $0.05)$ than those drawn from the lowest quartile $(\mu=0.40$, $\mathrm{SD}=0.13)$. Importantly, they also exhibited significantly higher 2nd phase CS responding compared to subjects from lowest quartile $(\mu=0.74, \mathrm{SD}=0.21 ; \mu=0.50, \mathrm{SD}=0.20$, respectively; $p$ 's $<0.05$ ), demonstrating that their greater responses for nicotine were consistent across cues. Following the same criteria for food-rewarded animals, we selected the highest quartile performing subjects on the 1 st phase $\mathrm{CR}$ test $(\mathrm{N}=8)$. A similar analysis procedure to that employed with the nicotine-rewarded animals was employed for food-rewarded animals, again finding a significant difference between high- $(\mu=0.92, \mathrm{SD}=0.05)$ and lowresponders $(\mu=0.65, \mathrm{SD}=0.12)$ in responding for the $1 \mathrm{st}$ phase CS; However, for this group, the $1^{\text {st }}$ phase-CS response differences were not matched by a significant difference in the 2 nd phase CS response for high- $(\mu=0.65$, $\mathrm{SD}=0.2)$ and low-responders $(\mu=0.66, \mathrm{SD}=0.21), \mathrm{p}>$ 0.05 .

Our final analysis repeated the initial 2 (blocked $v s$ nonblocked) $\times 2$ (food $v s$ drug) ANOVA comparing only those animals drawn from the highest quartile according to response to food or nicotine (Fig. 5). Repeated measures analyses revealed a significant decrement in CR performance among blocked food-rewarded animals between Phase I $(\mu=0.92, \mathrm{SD}=0.05)$ and Phase II $(\mu=0.66, \mathrm{SD}=0.13)$ $(\mathrm{F}(1,7)=16.40, \mathrm{p}<0.01)$, suggesting that blocking did take place in this group. When looking at the high-responding nicotine animals, no such decrement was found between Phase I $(\mu=0.91$, SD $=0.05)$, and Phase II $(\mu=0.74$, $\mathrm{SD}=0.21), \mathrm{F}(1,6)=4.33, \mathrm{p}>0.05$, a finding inconsistent with the blocking effect, and which seems to suggest that for this group, learning for the blocked CS was unimpeded.

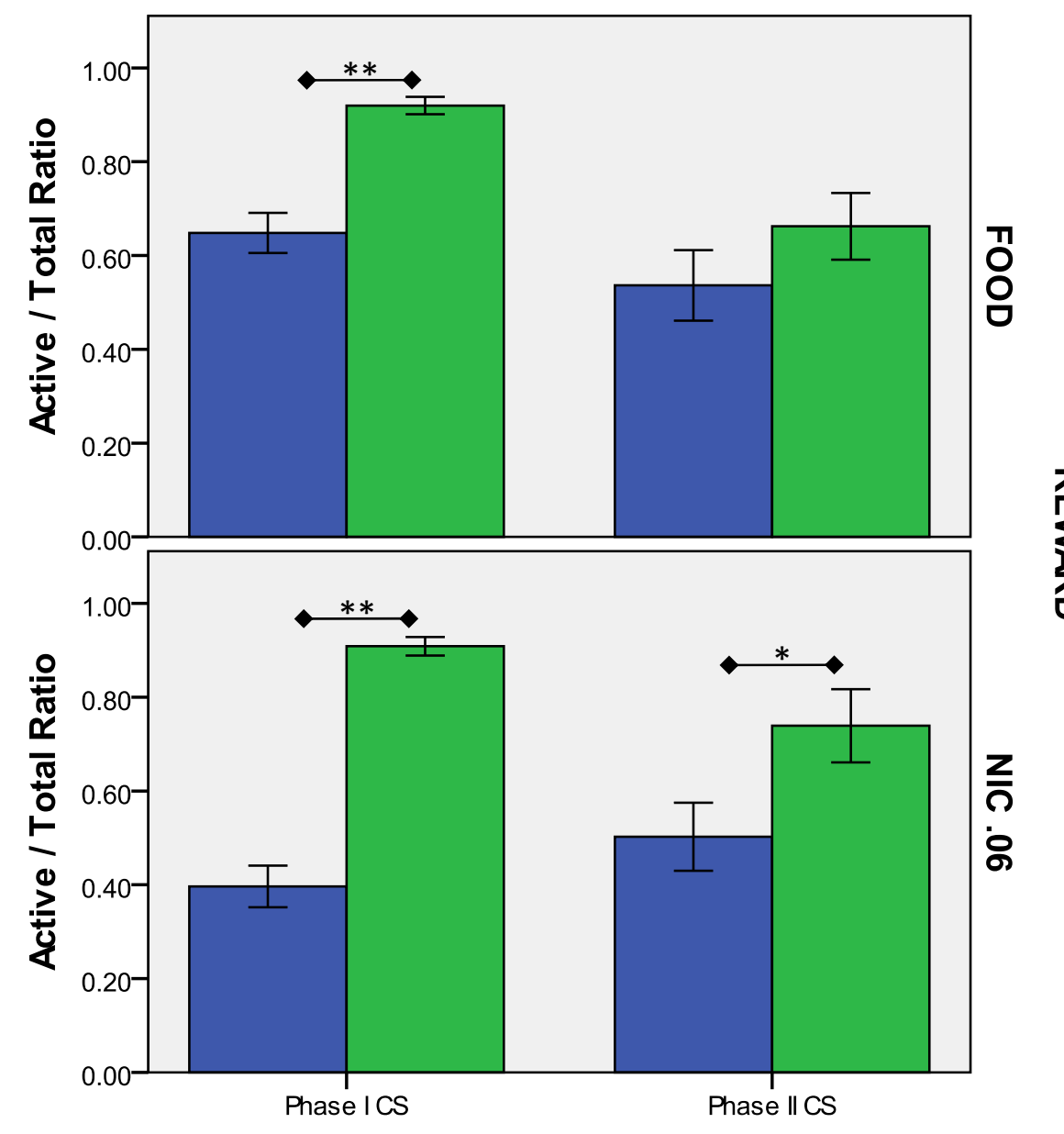

\section{Group}

Low Responders

High Responders

Error Bars: +/- $1 \mathrm{SE}$

Fig. (4). Performance in High vs Low Responding subjects on CR for both primary and secondary CS. 


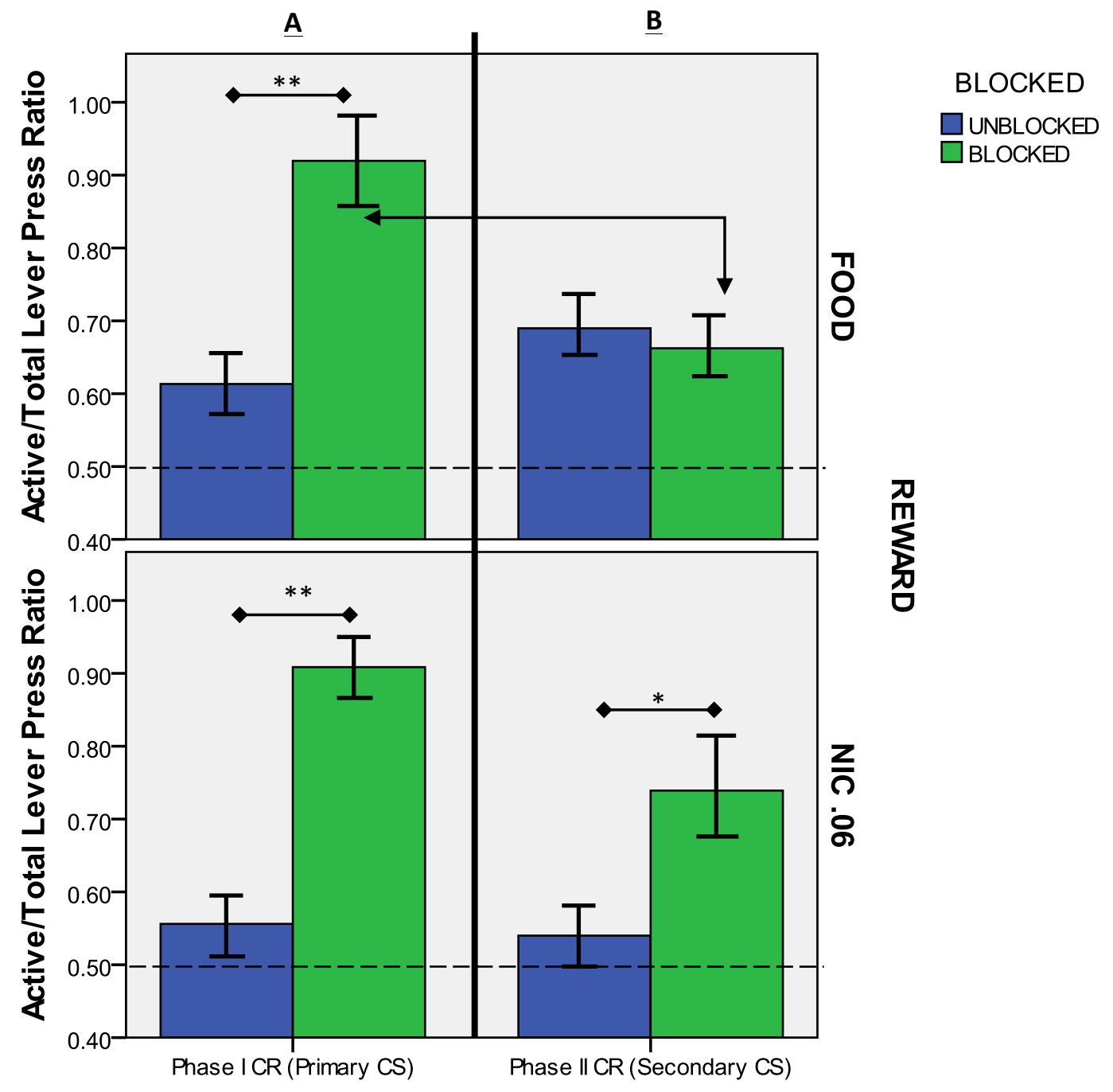

Note: The dashed line represents the point at which active presses = inactive presses $* p<0.05$

$* * p<0.01$

Fig. (5). Active-lever to total-press ratio for primary and secondary CR tests using only high-responding blocked subjects in both nicotine, and food, reward conditions.

\section{DISCUSSION}

Our goal in this experiment was to test the hypothesis that the use of nicotine as the reward in a Pavlovian conditioning blocking procedure would nullify the blocking effect and therefore fail to show reduced learning for a blocked $2^{\text {nd }}$ phase CS. Our finding support the hypothesis, with significant blocking found for food-, but not nicotinerewarded animals. However, while the initial hypothesis suggested increased learning for blocked cues among all drug-rewarded animals, our findings indicate that such an effect may be limited to high-responding subjects only. Such a distinction may not be surprising given the small effect found across all nicotine subjects, when compared with food, for conditioned reinforcement to the $1^{\text {st }}$ phase CS, an expected finding given previous work with nicotine $[9,10$, $39,40]$. Indeed, part of the motivation for this study was a resolution of the paradoxical finding that nicotine alone displays very little in the way of $1^{\text {st }}$ phase reinforcement effect and yet is one of the most commonly abused substances in the world. While a portion of this paradox may be explained by the enhancement of nicotine's 1st phasereinforcement value with the addition of monoamineoxidase-inhibitors and other chemicals commonly found in cigarette smoke [41-46] it seems likely that such effects require previous experience with nicotine [47, 48]. However, when our analysis focused on nicotine, and food, highresponders, Redish's (2004) hypothesis was robustly supported with significant learning shown for a $2^{\text {nd }}$ phase CS among blocked nicotine-rewarded animals but not foodrewarded animals and no evidence of blocking using repeated-measures analysis. Given the quantitative nature of the blocking effect on learning, namely attenuation in learning for blocked cues, these results indeed reveal no learning deficits for blocked cues among a subset of animals that show an increased response to nicotine.

The present findings raise the possibility that nicotine's $1^{\text {st }}$ phase-reinforcing value may be able to, through the nullification of the blocking effect, become associated with 
an exaggerated number of contextual cues, especially among those for whom the drug produces a robust reinforcing effect. Such an exaggerated distribution across cues could serve to indirectly enhance nicotine's reinforcement value and therefore the goal directing potential of nicotineassociated cues. Indeed, such an explanation could provide some resolution of the apparent nicotine-paradox presented earlier. However, while our findings seem to reveal such an effect for high-responder subjects, it is important to note the specificity of the effect, as it was not found among all subjects in the nicotine-reward condition. Such a distinction makes sense in the context of nicotine-dependence as not all those exposed to the drug find it rewarding and not all those who find it rewarding develop dependence.

The present study suffers from a number of limitations that limit its interpretation. Chief among these is the relatively small overall $\mathrm{CR}$ effect found for the 1st phase cues in the nicotine-reward condition. While such an effect is commonly found in studies using nicotine as a reward, future studies should examine whether the lifting of the blockingeffect is still present when other chemicals found in cigarette smoke are given in conjunction with nicotine. Such an extension would increase not only the generalizability of our findings, but would also improve the face-validity of the role of blocking in nicotine addiction. In order to resolve whether interference with the blocking effect, or a mere function of reduced exposure in Phase II, brought about the present findings, further studies should provide an increased exposure to Phase II conditioning procedures for nicotinerewarded animals. The exclusive use of male rats could also be considered a limitation, especially given the prevalence of smoking among women. Future investigations should include female animals in order to assure that the lifting of the blocking effect is not a gender-specific finding.

Tobacco addiction is one of the most destructive substance-abuse problems in the world due to its outsized prevalence and incredible impact on the health of users and non-users alike. Investigations into the mechanisms responsible for tobacco addiction are important for the development of appropriate interventions and prevention measures crucial for the stemming of tobacco's considerable impact on worldwide mortality and well-being. Previous work with nicotine revealed that it had a seemingly weak reinforcing effect and relied on contextual cues for its goal directing effect. Our findings suggest that within a particular subset of those exposed to the drug, nicotine's reinforcement value may, due to a lifting of the blocking effect, be conveyed to an exaggerated number of associated cues, when compared with natural rewards such as food, thereby biasing behavioral control towards it acquisition and possibly leading to the compulsive use patterns often reported among nicotine-dependent individuals.

\section{CONFLICT OF INTEREST}

The authors confirm that this article content has no conflict of interest.

\section{ACKNOWLEDGEMENTS} 02 .

Funding provided by NIDA grant number: DA025448-

\section{REFERENCES}

[1] Kamin LJ. Predictability, surprise, attention, and conditioning. In: Campbell BA, Church RM, Eds. Punishment and aversive behavior. New York: Appleton-Century-Crofts 1969; pp. 279-96.

[2] Benowitz NL. Clinical pharmacology of nicotine: Implications for understanding, preventing, and treating tobacco addiction. Clin Pharm Ther 2008; 83(4): 531-41.

[3] Mokdad AH, Marks JS, Stroup DF, Gerberding JL. Actual causes of death in the United States, 2000. J Am Med Assoc 2004; 291(10): 1238-45.

[4] Harvey DM, Yasar S, Heishman SJ, Panlilio LV, Henningfield JE, Goldberg SR. Nicotine serves as an effective reinforcer of intravenous drug-taking behavior in human cigarette smokers. Psychopharmacology 2004; 175(2): 134-42.

[5] Chen H, Matta SG, Sharp BM. Acquisition of nicotine selfadministration in adolescent rats given prolonged access to the drug. Neuropsychopharmacology 2007; 32(3): 700-9.

[6] Dadmarz M, Vogel WH. Individual self-administration of nicotine by rats. Pharmacol Biochem Behavior 2003; 76(3-4): 425-32.

[7] Henningfield JE, Goldberg SR. Nicotine as a reinforcer in human subjects and laboratory animals. Pharmacol Biochem Behavior 1983; 19(6): 989-92.

[8] Rose JE, Salley A, Behm FM, Bates JE, Westman EC. Reinforcing effects of nicotine and non-nicotine components of cigarette smoke. Psychopharmacology 2010; 210: 1-12.

[9] Caggiula AR, Donny EC, White AR, et al. Cue dependency of nicotine self-administration and smoking. Pharmacol Biochem Behav 2001; 70(4): 515-30.

[10] Caggiula AR, Donny EC, Chaudhri N, Perkins KA, Evans-Martin $\mathrm{FF}$, Sved AF. Importance of nonpharmacological factors in nicotine self-administration. Physiol Behav 2002; 77(4-5): 683-7.

[11] Chaudhri N, Caggiula AR, Donny EC, Palmatier MI, Liu X, Sved AF. Complex interactions between nicotine and nonpharmacological stimuli reveal multiple roles for nicotine in reinforcement. Psychopharmacology 2005; 184(3-4): 353-66.

[12] Cohen C, Perrault G, Griebel G, Soubrié P. Nicotine-associated cues maintain nicotine-seeking behavior in rats several weeks after nicotine withdrawal: reversal by the cannabinoid (CB1) receptor antagonist, rimonabant (SR141716). Neuropsychopharmacology 2004; 30(1): 145-55.

[13] Marchant HG, Moore JW. Blocking of the rabbit's conditioned nictitating membrane response in Kamin's two-stage paradigm. J Exp Psychol 1973; 101(1): 155-8.

[14] Shanks DR. Forward and backward blocking in human contingency judgement. Quart J Exp Psychol Section B 1985; 37(1): 1-21.

[15] Schultz W. Predictive reward signal of dopamine neurons. J Neurophysiol 1998; 80(1): 1-27.

[16] Redish AD. Addiction as a Computational Process Gone Awry. Science 2004; 306(5703): 1944-7.

[17] Culbertson C, Nicolas S, Zaharovits I, et al. Methamphetamine craving induced in an online virtual reality environment. Pharmacol Biochem Behav 2010; 96(4): 454-60.

[18] Liu X, Caggiula AR, Palmatier MI, Donny EC, Sved AF. Cueinduced reinstatement of nicotine-seeking behavior in rats: effect of bupropion, persistence over repeated tests, and its dependence on training dose. Psychopharmacology (Berl) 2008; 196(3): 365-75.

[19] Volkow ND, Wang GJ, Telang F, et al. Cocaine cues and dopamine in dorsal striatum: mechanism of craving in cocaine addiction. J Neuroscience 2006; 26(24): 6583-8.

[20] van de Laar MC, Licht R, Franken IH, Hendriks VM. Event-related potentials indicate motivational relevance of cocaine cues in abstinent cocaine addicts. Psychopharmacology (Berl) 2004; 177(1-2): 121-9.

[21] Grusser SM, Wrase J, Klein S, et al. Cue-induced activation of the striatum and medial prefrontal cortex is associated with subsequent relapse in abstinent alcoholics. Psychopharmacology (Berl) 2004; 175(3): 296-302.

[22] Kalivas PW, McFarland K. Brain circuitry and the reinstatement of cocaine-seeking behavior. Psychopharmacology (Berl) 2003; 168(1-2): 44-56.

[23] Panlilio LV, Thorndike EB, Schindler CW. Blocking of conditioning to a cocaine-paired stimulus: testing the hypothesis that cocaine perpetually produces a signal of larger-than-expected reward. Pharmacol Biochem Behavior 2007; 86(4): 774-7.

[24] Nisell M, Nomikos GG, Svensson TH. Systemic nicotine-induced dopamine release in the rat nucleus acccumbens is regulated by 
nicotinic receptors in the ventral tagmental area. Synapse 1994; 16 : 36-44.

[25] Andreoli M, Tessari M, Pilla M, Valerio E, Hagan JJ, Heidbreder CA. Selective antagonism at dopamine D3 receptors prevents nicotine-triggered relapse to nicotine-seeking behavior. Neuropsychopharmacology 2003; 28(7): 1272-80.

[26] Pontieri FE, Tanda G, Orzi F, Di Chiara G. Effects of nicotine on the nucleus accumbens and similarity to those of addictive drugs. Nature 1996; 382(6588): 255-7.

[27] Hollerman JR, Schultz W. Dopamine neurons report an error in the temporal prediction of reward during learning. Nature Neurosci 1998; 1(4): 304-9.

[28] Berridge $\mathrm{KC}$, Robinson TE. What is the role of dopamine in reward: Hedonic impact, reward learning, or incentive salience. Brain Res Rev 1998; 28(3): 309-69.

[29] Waelti P, Dickinson A, Schultz W. Dopamine responses comply with basic assumptions of formal learning theory. Nature 2001; 412(6842): 43-8.

[30] Wise RA. Dopamine, learning and motivation. Nature Rev Neurosci 2004; 5(6): 483-94.

[31] Sutton MA, Karanian DA, Self DW. Factors that determine a propensity for cocaine-seeking behavior during abstinence in rats. Neuropsychopharmacology 2000; 22(6): 626-41

[32] Pierre P, Vezina P. Predisposition to self-administer amphetamine: the contribution of response to novelty and prior exposure to the drug. Psychopharmacology 1997; 129(3): 277-84.

[33] Dalley J, Fryer T, Brichard L, et al. Nucleus accumbens D2/3 receptors predict trait impulsivity and cocaine reinforcement. Science 2007; 315(5816): 1267

[34] Perry J, Larson E, German J, Madden G, Carroll M. Impulsivity (delay discounting) as a predictor of acquisition of IV cocaine selfadministration in female rats. Psychopharmacology 2005; 178(2): 193-201.

[35] Pianezza M, Sellers E, Tyndale R. Nicotine metabolism defect reduces smoking. Nature 1998; 393(6687): 750.

[36] Tyndale R, Sellers E. Genetic variation in CYP2A6-mediated nicotine metabolism alters smoking behavior. Therap Drug Monitor 2002; 24(1): 163.
[37] Shram MJ, Funk D, Li Z, Le AD. Nicotine Self-Administration, Extinction Responding and Reinstatement in Adolescent and Adult Male Rats: Evidence Against a Biological Vulnerability to Nicotine Addiction during Adolescence. Neuropsychopharmacology 2007; 33(4): 739-48.

[38] Shoaib M, Stolerman IP. Plasma nicotine and cotinine levels following intravenous nicotine self-administration in rats. Psychopharmacology (Berl) 1999; 143(3): 318-21.

[39] Palmatier MI, Evans-Martin FF, Hoffman A, et al. Dissociating the primary reinforcing and reinforcement-enhancing effects of nicotine using a rat self-administration paradigm with concurrently available drug and environmental reinforcers. Psychopharmacology (Berl). 2006; 184(3-4): 391-400.

[40] Chaudhri N, Caggiula AR, Donny EC, Palmatier MI, Liu X, Sved AF. Complex interactions between nicotine and nonpharmacological stimuli reveal multiple roles for nicotine in reinforcement. Psychopharmacology 2005; 184: 353-66.

[41] Agatsuma S, Lee M, Zhu H, et al. Monoamine oxidase A knockout mice exhibit impaired nicotine preference but normal responses to novel stimuli. Human Molr Genetics 2006; 15(18): 2721-31.

[42] Guillem K, Vouillac C, Azar M, et al. Monoamine oxidase inhibition dramatically increases the motivation to self-administer nicotine in rats. J Neurosci 2005; 25(38): 8593-600.

[43] Leroy C, Bragulat V, Berlin I, et al. Cerebral monoamine oxidase A inhibition in tobacco smokers confirmed with PET and [11C] befloxatone. J Clin Psychopharmacol 2009; 29(1): 86-8.

[44] Lewis A, Miller J, Lea R. Monoamine oxidase and tobacco dependence. Neurotoxicology 2007; 28(1): 182-95.

[45] Stephenson J. Clues found to tobacco addiction. J Am Med Assoc 1996; 275(16): 1217-8

[46] Talhout R, Opperhuizen A, van Amsterdam J. Role of acetaldehyde in tobacco smoke addiction. Eur J Neuropsychopharmacol 2007; 17(10): 627-36.

[47] Fowler J, Wang G, Volkow N, et al. Smoking a single cigarette does not produce a measurable reduction in brain MAO B in nonsmokers. Nicotine Tobacco Res 1999; 1(4): 325-9.

[48] Guillem K, Vouillac C, Azar M, et al. Monoamine oxidase A rather than monoamine oxidase $\mathrm{B}$ inhibition increases nicotine reinforcement in rats. Eur J Neurosci 2006; 24(12): 3532-40. 